\title{
Revisão
}

\section{Impasses e desafios para consolidação e efetividade do apoio matricial em saúde mental no Brasil}

The consolidation and effectiveness of matrix support in mental health in Brazil - bottlenecks and challenges (abstract: p. 18)

Impases y desafíos para consolidación y efectividad del apoyo matricial en salud mental en Brasil (resumen: p. 18)

Carlos Alberto dos Santos Treichel ${ }^{(a)}$

$<$ carlos-treichel@hotmail.com>

Rosana Teresa Onocko Campos ${ }^{(b)}$

<rosanaoc@mpc.com.br>

Gastão Wagner de Souza Campos ${ }^{(\mathrm{c})}$

<gastaowagner@mpc.com.br> (iD) (a) Pós-graduando do programa de Pós-Graduação em Saúde Coletiva (Doutorado), Departamento de Saúde Coletiva, Faculdade de Ciências Médicas, Universidade Estadual de Campinas (Unicamp). Rua Tessália Vieira de Camargo, 126, Cidade Universitária Zeferino Vaz. Campinas, SP, Brasil. 13083887.

$(b, c)$ Departamento de Saúde Coletiva, Faculdade de Ciências Médicas, Unicamp. Campinas, SP, Brasil.

Passados dez anos da implementação do apoio matricial nas redes de saúde por meio dos Núcleos de Apoio à Saúde da Família, ainda sente-se a falta de dados consistentes quanto à consolidação e efetividade desse arranjo. Nesse sentido, este estudo teve como objetivo revisar a bibliografia nacional dos últimos dez anos a fim de identificar os impasses e desafios vivenciados no apoio matricial em saúde mental na Atenção Primária, classificando-os a partir de uma reconstrução teórico-conceitual e fazendo uma articulação destes com os desafios pontuados em congêneres internacionais do apoio matricial. Entre os principais pontos levantados pelo estudo, destacou-se a necessidade de delineamentos claros para prática de matriciamento; investimento maciço em formação e capacitação dos profissionais; e criação de espaços institucionalizados com encontros sistemáticos dos profissionais para discussão dos casos e avaliação conjunta do andamento das atividades.

Palavras-chave: Apoio matricial. Saúde mental. Atenção primária à saúde. 


\section{Introdução}

A provisão de cuidados de qualidade aos usuários com problemas de saúde mental constitui um dos grandes desafios para os sistemas de saúde em todo o mundo.

Aspectos como taxas de mortalidade duas a três vezes maiores em relação à população geral e uma expectativa de vida reduzida entre dez e trinta anos ${ }^{1}$ levaram a Organização Mundial da Saúde a pontuar a superação dessas disparidades como um dos desafios do milênio².

Entre os fatores contributivos para o estabelecimento desse cenário, destacamse a baixa integração das redes de saúde e a falta de profissionais preparados para providenciar cuidados adequados em saúde mental na Atenção Primária ${ }^{3,4}$. Como desdobramentos desse cenário, há uma baixa capacidade de identificação e manejo dos casos nos níveis primários de atenção, sobrecarga dos serviços especializados, e consequentemente, dificuldade de acesso aos serviços de saúde mental em tempo oportuno $^{5-8}$.

Ao redor do mundo, uma das estratégias utilizadas a fim de superar esse desafio é a implementação de dispositivos de integração de rede que visem fomentar a participação conjunta entre profissionais de Atenção Primária e especialistas. Destacam-se iniciativas realizadas em países como Austrália e Canadá, tais como cuidado compartilhado (shared care) e cuidado colaborativo (collaborative care), ${ }^{9}$.

No Brasil, como congênere dessas iniciativas, surge a proposta de apoio matricial, que visa ampliar as possibilidades de um cuidado integral e da integração dialógica entre distintas especialidades e profissóes. Trata-se de um modelo de intervenção pedagógico-terapêutica que visa produzir e estimular padróes de relação que perpassem todos trabalhadores e usuários, favorecendo a troca de informações e a ampliação da corresponsabilização pelo usuário ${ }^{11}$. Destaca-se ainda a atuação das equipes de apoio matricial como uma retaguarda especializada de assistência, evitando, dessa forma, os encaminhamentos desnecessários a outros níveis de atendimento e aumentando a capacidade resolutiva de problemas de saúde pela equipe de referência.

Ressalta-se que essa estratégia de cogestão para a organização do trabalho interprofissional foi formulada no início da década de 1990 e passou a ser implementada por iniciativa dos profissionais da rede do Sistema Único de Saúde (SUS) de Campinas-SP na área da Saúde Mental. Tal aspecto talvez seja um dos grandes contribuidores para a consolidação de certa tradição do apoio matricial nessa área, embora, com o decorrer dos anos, tenha havido uma expansão para outras áreas de saber especializado como a Reabilitação Física, a Traumatologia, a Dermatologia, entre outras $^{12}$.

Como principal marco do processo de incorporação do apoio matricial nas redes de saúde, destaca-se a proposta ministerial de criação dos Núcleos de Apoio à Saúde da Família (Nasf), por meio da portaria no 154 , de 24 de janeiro de $2008^{13}$. A esses serviços, conferiu-se o papel de retaguarda clínico-assistencial e apoio técnicopedagógico, institucionalizando assim o apoio matricial como ferramenta de trabalho na Saúde da Família.

Passados dez anos da implementação dos Nasf, cabe ressaltar que o número de serviços desse tipo aumentou exponencialmente. Enquanto existiam três Nasf no ano de 2008, de acordo com os dados do departamento de Atenção Básica (AB) do Ministério da Saúde, em outubro de 2018 esse número já chegava a 5090². Dessa 
forma, hoje o Nasf representa a maior aposta de um dispositivo de integração de rede, capaz de fomentar a participação conjunta entre profissionais de Atenção Básica e especialistas de Saúde Mental no país.

Contudo, parece haver ainda algumas limitações no que tange a consolidação e efetividade desses serviços como mediadores do atendimento em Saúde Mental na Atenção Básica, havendo necessidade de avaliar quais são os impasses e desafios que têm dificultado esses processos.

No ano anterior à promulgação da portaria no $154 / 2008$, Campos e Domitti ${ }^{15}$ publicaram uma reconstrução teórico-conceitual da metodologia de gestão do trabalho em saúde baseada em equipes de referência e apoio matricial como base para apoiar pesquisas que avaliassem limitaçóes e potências dessa modalidade organizacional. Dentre os desafios antecipados pelos autores, destacavam-se obstáculos estruturais, éticos, políticos, culturais, epistemológicos e subjetivos ao desenvolvimento desse tipo de trabalho integrado em saúde.

Nesse sentido, este estudo teve como objetivo revisar a bibliografia nacional dos últimos dez anos a fim de identificar os impasses e desafios para consolidação e efetividade do apoio matricial em Saúde Mental na Atenção Primária por meio dos Nasf, classificando-os a partir da reconstrução teórico-conceitual realizada por Campos e Domitti ${ }^{15}$ e fazendo uma articulação destes com os desafios pontuados em congêneres internacionais do apoio matricial.

\section{Metodologia}

A fim de identificar os impasses e desafios para consolidação e efetividade do apoio matricial em Saúde Mental na Atenção Básica por meio dos Nasf, foi realizada uma revisão bibliográfica integrativa. A busca visou captar estudos que tivessem trabalhado com os atores implicados nas açóes desses serviços - usuários, trabalhadores e gestores - e foi orientada pela seguinte questão norteadora: quais os impasses e desafios vivenciados no apoio matricial em Saúde Mental?

A busca ocorreu no mês de agosto de 2018 e rastreou estudos publicados nos últimos dez anos - 2008 a 2018 - realizados no contexto brasileiro. As bases de dados utilizadas para buscar estudos foram PubMed (Publisher Medline) e SciELO. org (Scientific Electronic Library Online). Os termos utilizados para a busca foram: Atenção Primária à Saúde; Programa de Saúde da Família; Estratégia de Saúde da Família; Nasf e Apoio Matricial. Para a busca, todos os termos foram traduzidos para língua inglesa, a saber: Primary Health Care; Family Health Program; Family Health Strategy; Nasf e Matrix Support. Os filtros considerados na busca foram: "estudos publicados nos últimos dez anos" e "publicados em língua inglesa", "portuguesa” ou "espanhola”.

Dado o critério de inclusão "estudos conduzidos com usuários, trabalhadores ou gestores dos serviços da Atenção Primária ou Nasf”, todos os artigos rastreados foram avaliados primeiramente por seus títulos e resumos. Nessa etapa, foram excluídos os estudos que não se encaixavam no escopo desta revisão ou se constituíam como revisóes de literatura e reflexôes teóricas. Todos os artigos que atenderam os critérios de inclusão ou não apresentavam elementos suficientes para determinar sua exclusão foram obtidos na íntegra e avaliados de acordo com o seguinte critério de exclusão: 
Não pontuar quais os impasses e/ou desafios para consolidação e efetividade do apoio matricial em Saúde Mental na Atenção Primária por meio dos Nasf.

Optou-se por realizar a categorização dos desafios e impasses identificados nos estudos rastreados de acordo com os tópicos sugeridos por Campos e Domitti ${ }^{15} \mathrm{em}$ sua reconstrução teórico-conceitual da metodologia de gestão do trabalho em saúde baseada em equipes de referência e apoio matricial, a saber: obstáculos estruturais, éticos, políticos, culturais, epistemológicos e subjetivos.

Para essa categorização, os autores se orientaram pelo modelo de análise de conteúdo categorial temático de Minayo ${ }^{16}$. Ou seja, a análise foi conduzida em etapas, sendo a primeira relacionada às operações de desmembramento dos textos em unidades e em categorias e a segunda relacionada ao reagrupamento analítico por meio da organização das mensagens a partir dos elementos repartidos. Para esse segundo momento, cada item foi discutido a fim de estabelecer um consenso quanto à sua alocação nos tópicos propostos.

Os aspectos éticos foram atendidos neste estudo, na medida em que as informações e as ideias dos autores foram respeitadas, assegurando-se autoria e citação nas referências.

\section{Resultados}

Um total de 595 estudos foram rastreados nas buscas, sendo 233 da SciELO e 362 da PubMed. Destes, 493 estudos foram descartados por estarem fora da temática proposta por essa revisão. Outros 43 estudos foram descartados por estarem duplicados, sete por se tratarem de revisóes de literatura, cinco por serem reflexóes teóricas e dois por serem resumos de tese. Dessa forma, foram obtidos 45 estudos para leitura na íntegra, dos quais sete foram descartados por não responderem a questão norteadora da busca, restando 38 estudos que foram incluídos nesta revisão. A figura 1 apresenta por meio de fluxograma o percurso para seleção dos artigos.

Os estudos selecionados podem ser observados no quadro 1 , no qual estão caracterizados por seus títulos, autores, local de realização, população estudada, tipo de abordagem do estudo e ano de publicação, além de código próprio atribuído a fim de facilitar sua identificação no agrupamento por categorias. 
(2)

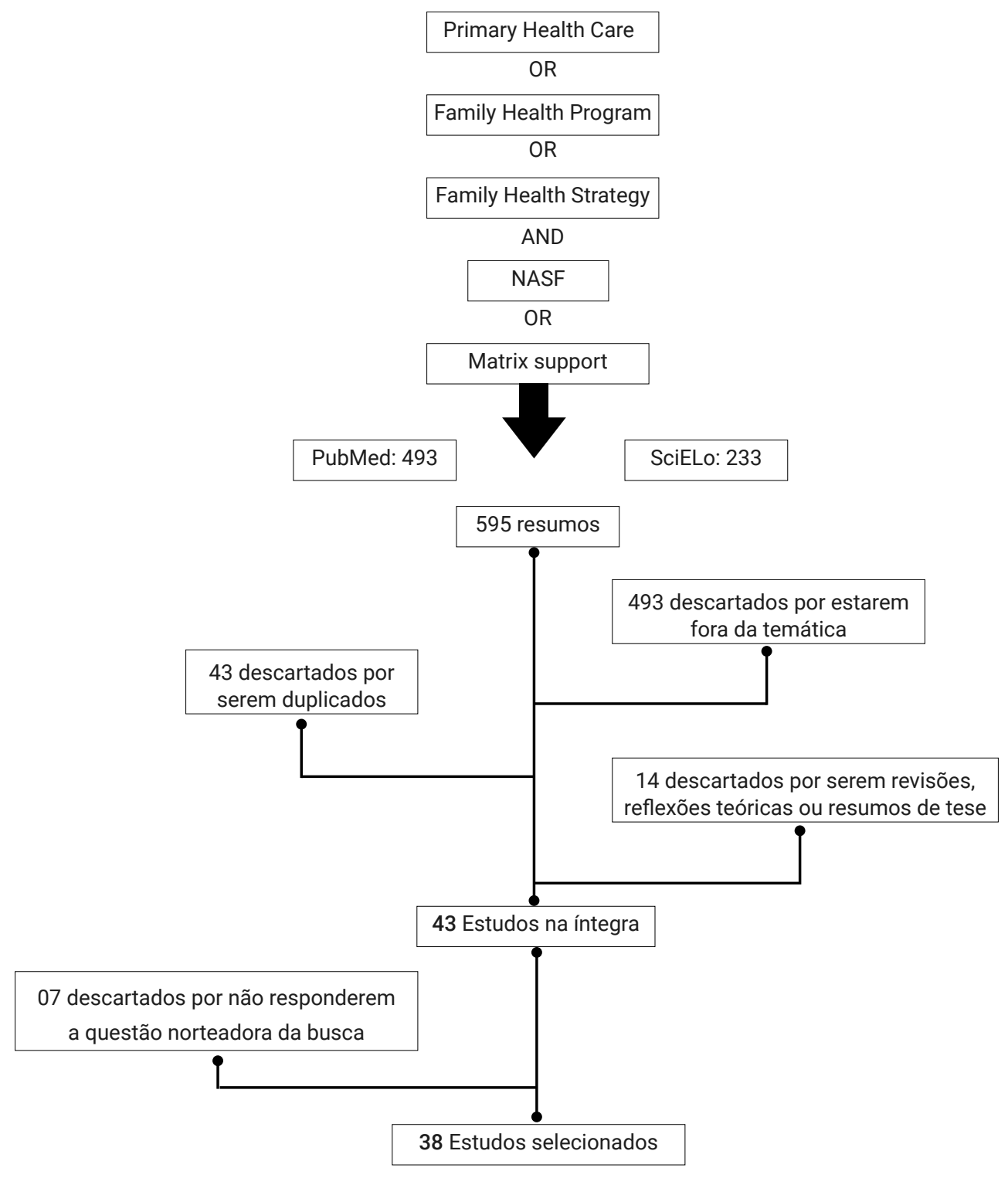

Figura 1. Fluxograma da busca, exclusão e seleção dos artigos

Quadro 1. Relação dos estudos selecionados de acordo com seus títulos, autores, local de realização, população, tipo de abordagem e ano de publicação

\begin{tabular}{|c|c|c|c|c|c|c|}
\hline :ं் & Título & Autores & Local & População & Abordagem & Ano \\
\hline $\bar{\square}$ & $\begin{array}{l}\text { O Apoio Matricial em Unidades de Saúde } \\
\text { da Família: experimentando inovações em } \\
\text { saúde mental }\end{array}$ & $\begin{array}{l}\text { Dimenstein et } \\
\text { al }^{17}\end{array}$ & São Paulo-SP & 8 trabalhadores da AB & Qualitativa & 2009 \\
\hline Ш & $\begin{array}{c}\text { Saúde Mental na atenção básica à saúde } \\
\text { de Campinas, SP: uma rede ou um } \\
\text { emaranhado? }\end{array}$ & $\begin{array}{l}\text { Figueiredo } \\
\text { e Onocko- } \\
\text { Campos }^{18}\end{array}$ & Campinas-SP & $\begin{array}{l}16 \text { trabalhadores } \\
\text { da AB e serviços } \\
\text { especializados }\end{array}$ & Qualitativa & 2009 \\
\hline$\ddot{\Psi}$ & $\begin{array}{l}\text { Saúde mental na Estratégia Saúde da } \\
\text { Família: a avaliação de apoio matricial }\end{array}$ & $\begin{array}{c}\text { Mielke e } \\
\text { Olchowsky }{ }^{19}\end{array}$ & Porto Alegre-RS & 14 profissionais da $A B$ & Qualitativa & 2010 \\
\hline
\end{tabular}


Quadro 1. Relação dos estudos selecionados de acordo com seus títulos, autores, local de realização, população, tipo de abordagem e ano de publicação

\begin{tabular}{|c|c|c|c|c|c|c|}
\hline : & Título & Autores & Local & População & Abordagem & Ano \\
\hline 岀 & $\begin{array}{c}\text { Saúde mental na atenção primária à saúde: } \\
\text { estudo avaliativo em uma grande cidade } \\
\text { brasileira }\end{array}$ & $\begin{array}{l}\text { Onocko- } \\
\text { Campos et } \mathrm{al}^{5}\end{array}$ & Campinas-SP & $\begin{array}{l}65 \text { usuários e } 72 \\
\text { trabalhadores da } A B\end{array}$ & Qualitativa & 2011 \\
\hline 㠻 & $\begin{array}{l}\text { Avaliação das ações em saúde mental } \\
\text { na Estratégia de Saúde da Família: } \\
\text { necessidades e potencialidades }\end{array}$ & $\begin{array}{l}\text { Cossetin e } \\
\text { Olschowsky }{ }^{20}\end{array}$ & Porto Alegre-RS & $\begin{array}{l}8 \text { participantes de } \\
\text { conselhos locais de } \\
\text { saúde }\end{array}$ & Qualitativa & 2011 \\
\hline 岀 & $\begin{array}{l}\text { Centro de Atenção Psicossocial: } \\
\text { convergência entre Saúde Mental e Coletiva }\end{array}$ & Ballarin et $\mathrm{al}^{21}$ & Campinas-SP & $\begin{array}{l}7 \text { trabalhadores de um } \\
\text { Caps }\end{array}$ & Qualitativa & 2011 \\
\hline Ш & $\begin{array}{c}\text { Apoio matricial em saúde mental: uma } \\
\text { análise sob ótica dos profissionais de saúde } \\
\text { da atenção primária }\end{array}$ & Silva ${ }^{22}$ & Aracaju-SE & $\begin{array}{l}15 \text { trabalhadores de um } \\
\text { Caps }\end{array}$ & Qualitativa & 2011 \\
\hline$\stackrel{\infty}{\Psi}$ & $\begin{array}{l}\text { Tecendo a rede assistencial em saúde } \\
\text { mental com a ferramenta matricial }\end{array}$ & Sousa et $\mathrm{al}^{23}$ & $\begin{array}{l}\text { Dois municípios } \\
\text { situados na } \\
\text { região nordeste }\end{array}$ & $\begin{array}{l}47 \text { trabalhadores de } \\
\text { Caps e da } A B\end{array}$ & Qualitativa & 2011 \\
\hline யి & $\begin{array}{l}\text { Avaliação de estratégias inovadoras na } \\
\text { organização da Atenção Primária à Saúde }\end{array}$ & $\begin{array}{l}\text { Onocko- } \\
\text { Campos et al }\end{array}$ & Campinas-SP & $\begin{array}{l}65 \text { usuários e } 72 \\
\text { trabalhadores da } A B\end{array}$ & Qualitativa & 2012 \\
\hline 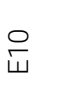 & $\begin{array}{l}\text { Apoio matricial: um estudo sobre a } \\
\text { perspectiva de profissionais da saúde } \\
\text { mental }\end{array}$ & Ballarin et $\mathrm{al}^{24}$ & Campinas-SP & $\begin{array}{l}14 \text { trabalhadores de } \\
\text { Caps e da } A B\end{array}$ & Qualitativa & 2012 \\
\hline $\bar{\varpi}$ & $\begin{array}{c}\text { Onde está a criança? Desafios e obstáculos } \\
\text { ao apoio matricial de crianças com } \\
\text { problemas de saúde mental }\end{array}$ & $\begin{array}{l}\text { Cavalcante et } \\
\qquad \mathrm{a}^{25}\end{array}$ & Fortaleza-CE & $\begin{array}{l}2 \text { trabalhadores da } A B \text { e } \\
4 \text { familiares de crianças } \\
\text { cujos casos passaram } \\
\text { por matriciamento }\end{array}$ & Qualitativa & 2012 \\
\hline$\stackrel{\simeq}{\square}$ & $\begin{array}{l}\text { Ferramenta matricial na produção do } \\
\text { cuidado integral na estratégia saúde da } \\
\text { família }\end{array}$ & Jorge et $a^{26}$ & $\begin{array}{l}\text { Dois municípios } \\
\text { situados no } \\
\text { estado do Ceará }\end{array}$ & $\begin{array}{c}55 \text { trabalhadores } \\
\text { da rede de SM, } 25 \\
\text { usuários atendidos } \\
\text { pelo matriciamento e } \\
11 \text { familiares desses } \\
\text { usuários }\end{array}$ & Qualitativa & 2012 \\
\hline$\frac{m}{\square}$ & $\begin{array}{c}\text { Articulação entre serviços públicos de saúde } \\
\text { nos cuidados voltados à saúde mental } \\
\text { infantojuvenil. }\end{array}$ & Delfini e Reis ${ }^{27}$ & São Paulo-SP & $\begin{array}{l}18 \text { trabalhadores de } \\
\text { CAPS-IJ e da AB }\end{array}$ & Qualitativa & 2012 \\
\hline$\stackrel{⿱}{\dot{\Xi}}$ & $\begin{array}{l}\text { Apoio Matricial em Saúde Mental: alcances e } \\
\text { limites na atenção básica }\end{array}$ & $\begin{array}{l}\text { Morais e } \\
\text { Tanaka }^{28}\end{array}$ & Fortaleza-CE & 12 trabalhadores da $A B$ & Qualitativa & 2012 \\
\hline $\begin{array}{l}\llcorner \\
\dot{\square}\end{array}$ & $\begin{array}{l}\text { A interlocução da Saúde Mental com } \\
\text { Atenção Básica no município de Vitória-ES }\end{array}$ & $\begin{array}{l}\text { Rodrigues e } \\
\text { Moreira }^{29}\end{array}$ & Vitória-ES & $\begin{array}{l}14 \text { trabalhadores de } \\
\text { Caps e da } A B\end{array}$ & Qualitativa & 2012 \\
\hline$\frac{0}{\dot{w}}$ & $\begin{array}{l}\text { Modelos tecnoassistenciais e atuação do } \\
\text { psiquiatra no campo da atenção primária à } \\
\text { saúde no contexto atual do Sistema Único } \\
\text { de Saúde, Brasil }\end{array}$ & $\begin{array}{l}\text { Vannucchi } \\
\text { e Carneiro- } \\
\text { Junior }{ }^{30}\end{array}$ & São Paulo-SP & $\begin{array}{c}2 \text { médicos psiquiatras } \\
\text { vinculados à } A B \text { e a um } \\
\text { Nasf }\end{array}$ & Qualitativa & 2012 \\
\hline$\stackrel{\wedge}{\bar{w}}$ & $\begin{array}{l}\text { O psicólogo apoiador matricial: percepções } \\
\text { e práticas na Atenção Básica }\end{array}$ & $\begin{array}{l}\text { Freire e } \\
\text { Pichelli¹ }\end{array}$ & $\begin{array}{l}\text { João Pessoa- } \\
\text { PB }\end{array}$ & $\begin{array}{l}10 \text { psicólogos } \\
\text { vinculados ao Nasf }\end{array}$ & Qualitativa & 2013 \\
\hline$\stackrel{\infty}{\square}$ & $\begin{array}{c}\text { Apoio matricial em saúde mental: } \\
\text { fortalecendo a saúde da família na clínica } \\
\text { da crise }\end{array}$ & $\begin{array}{l}\text { Minozzo e } \\
\text { Costa }^{32}\end{array}$ & $\begin{array}{l}\text { Rio de Janeiro- } \\
\text { RJ }\end{array}$ & $\begin{array}{l}6 \text { trabalhadores da } A B \\
\text { e } 15 \text { trabalhadores de } \\
\text { Caps }\end{array}$ & Qualitativa & 2013 \\
\hline$\frac{\varrho}{\square}$ & $\begin{array}{l}\text { A inserção da Psicologia nos Núcleos de } \\
\text { Apoio à Saúde da Família }\end{array}$ & Leite et al ${ }^{33}$ & $\begin{array}{l}\text { Juazeiro do } \\
\text { Norte-CE }\end{array}$ & $\begin{array}{l}6 \text { psicólogos vinculados } \\
\text { a um Nasf }\end{array}$ & Qualitativa & 2013 \\
\hline ิㅗ & $\begin{array}{c}\text { Acessibilidade e resolubilidade da } \\
\text { assistência em saúde mental: a experiência } \\
\text { do apoio matricial }\end{array}$ & Quinderé et al ${ }^{34}$ & $\begin{array}{l}\text { Fortaleza-CE e } \\
\text { Sobral-CE }\end{array}$ & $\begin{array}{c}37 \text { trabalhadores, } \\
14 \text { usuários e } 13 \\
\text { familiares da AB cujos } \\
\text { casos passaram por } \\
\text { matriciamento }\end{array}$ & Qualitativa & 2013 \\
\hline$\overline{\mathrm{N}}$ & $\begin{array}{c}\text { Apoio matricial em saúde mental entre Caps } \\
\text { e Saúde da Família: trilhando caminhos } \\
\text { possíveis }\end{array}$ & $\begin{array}{l}\text { Minozzo e } \\
\text { Costa }^{35}\end{array}$ & $\begin{array}{l}\text { Rio de Janeiro- } \\
\text { RJ }\end{array}$ & $\begin{array}{c}15 \text { trabalhadores de } \\
\text { Caps e } 5 \text { trabalhadores } \\
\text { da AB }\end{array}$ & Qualitativa & 2013 \\
\hline
\end{tabular}


Quadro 1. Relação dos estudos selecionados de acordo com seus títulos, autores, local de realização, população, tipo de abordagem e ano de publicação

\begin{tabular}{|c|c|c|c|c|c|c|}
\hline 'ீं & Título & Autores & Local & População & Abordagem & Ano \\
\hline ญิ & $\begin{array}{c}\text { Apoio matricial: dispositivo para resolução } \\
\text { de casos clínicos de saúde mental na } \\
\text { Atenção Primária à Saúde }\end{array}$ & Jorge et al ${ }^{36}$ & Fortaleza-CE & $\begin{array}{l}3 \text { trabalhadores da } \mathrm{AB} \text { e } \\
3 \text { trabalhadores do Caps }\end{array}$ & Qualitativa & 2013 \\
\hline$\stackrel{\mathscr{N}}{\Psi}$ & $\begin{array}{l}\text { Saúde mental na atenção básica: o trabalho } \\
\text { em rede e o matriciamento em saúde mental } \\
\text { na Estratégia de Saúde da Família }\end{array}$ & $\begin{array}{l}\text { Gazignato e } \\
\quad \text { Silva }^{37}\end{array}$ & Guarujá-SP & 10 trabalhadores da $\mathrm{AB}$ & Qualitativa & 2014 \\
\hline$\stackrel{\mathbb{\Psi}}{\uplus}$ & $\begin{array}{c}\text { O Projeto Terapêutico Singular e as práticas } \\
\text { de saúde mental nos Núcleos de Apoio à } \\
\text { Saúde da Família (Nasf) em Guarulhos (SP), } \\
\text { Brasil. }\end{array}$ & $\begin{array}{c}\text { Hori e } \\
\text { Nascimento }{ }^{38}\end{array}$ & Guarulhos-SP & $\begin{array}{l}13 \text { trabalhadores de } \\
\text { Nasf }\end{array}$ & Mista & 2014 \\
\hline$\stackrel{\sim}{\mathbb{W}}$ & $\begin{array}{c}\text { Matriciamento em Saúde Mental segundo } \\
\text { profissionais da Estratégia da Saúde da } \\
\text { Família }\end{array}$ & Pegoraro et al ${ }^{39}$ & Goiania-GO & 12 trabalhadores da $\mathrm{AB}$ & Mista & 2014 \\
\hline$\stackrel{\sim}{\mathbb{w}}$ & $\begin{array}{c}\text { Desafios do apoio matricial como prática } \\
\text { educacional: a saúde mental na atenção } \\
\text { básica }\end{array}$ & Costa et al ${ }^{40}$ & São Carlos-SP & $\begin{array}{l}18 \text { trabalhadores de } \\
\text { matriciamento. }\end{array}$ & Qualitativa & 2015 \\
\hline ๙ิ & $\begin{array}{l}\text { Apoio Matricial, Projeto Terapêutico Singular } \\
\text { e Produção do Cuidado em Saúde Mental }\end{array}$ & Jorge et $\mathrm{a}^{41}$ & Fortaleza-CE & $\begin{array}{l}17 \text { trabalhadores de } \mathrm{AB} \\
\text { Caps e Nasf }\end{array}$ & Qualitativa & 2015 \\
\hline $\begin{array}{l}\stackrel{\infty}{\sim} \\
w\end{array}$ & $\begin{array}{c}\text { A perspectiva dos profissionais da Atenção } \\
\text { Primária à Saúde sobre o apoio matricial em } \\
\text { saúde mental }\end{array}$ & Hirdes $^{7}$ & Porto Alegre-RS & $\begin{array}{l}8 \text { trabalhadores de } \\
\text { matriciamento }\end{array}$ & Qualitativa & 2015 \\
\hline ญิ & $\begin{array}{l}\text { As contribuições dos psicólogos para o } \\
\text { Matriciamento em Saúde Mental. }\end{array}$ & $\begin{array}{l}\text { Iglesias e } \\
\text { Avellar }{ }^{42}\end{array}$ & $\begin{array}{l}\text { Um município } \\
\text { do Espírito } \\
\text { Santo }\end{array}$ & 6 trabalhadores da $\mathrm{AB}$ & Qualitativa & 2016 \\
\hline 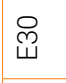 & $\begin{array}{l}\text { Avaliação do trabalho multiprofissional do } \\
\text { Núcleo de Apoio à Saúde da Família (Nasf) }\end{array}$ & Reis et $\mathrm{al}^{43}$ & Florianópolis-SC & $\begin{array}{l}14 \text { trabalhadores de } \\
\text { Nasf }\end{array}$ & Qualitativa & 2016 \\
\hline$\overline{\tilde{m}}$ & $\begin{array}{c}\text { Apoio Matricial e Capsi: desafios do cenário } \\
\text { na implantação do matriciamento em saúde } \\
\text { mental }\end{array}$ & Salvador e Pio ${ }^{44}$ & Marília-SP & $\begin{array}{l}9 \text { trabalhadores de } \\
\text { CAPS-IJ. }\end{array}$ & Qualitativa & 2016 \\
\hline$\stackrel{\widetilde{W}}{\tilde{W}}$ & $\begin{array}{l}\text { O apoio matricial em saúde mental: uma } \\
\text { ferramenta apoiadora da atenção à crise }\end{array}$ & $\begin{array}{c}\text { Lima e } \\
\text { Dimenstein }{ }^{45}\end{array}$ & Recife-PE & $\begin{array}{l}27 \text { trabalhadores de } \\
\text { Caps }\end{array}$ & Qualitativa & 2016 \\
\hline$\stackrel{\mathscr{m}}{\Psi}$ & $\begin{array}{l}\text { The matrix approach to mental health care: } \\
\text { experiences in Florianopolis, Brazil }\end{array}$ & $\begin{array}{l}\text { Soares e } \\
\text { Oliveira }{ }^{46}\end{array}$ & Florianópolis-SC & 6 trabalhadores de Nasf & Qualitativa & 2016 \\
\hline 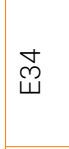 & $\begin{array}{l}\text { Perceptions of health managers and } \\
\text { professionals about mental health and } \\
\text { primary care integration in Rio de Janeiro: a } \\
\text { mixed methods study }\end{array}$ & Athié et al ${ }^{8}$ & $\begin{array}{l}\text { Rio de Janeiro- } \\
\text { RJ }\end{array}$ & $\begin{array}{l}42 \text { trabalhadores de } \\
\text { Nasf }\end{array}$ & Mista & 2016 \\
\hline$\stackrel{\llcorner}{\tilde{m}}$ & $\begin{array}{c}\text { O "cabo de força” da assistência: concepção } \\
\text { e prática de psicólogos sobre o Apoio } \\
\text { Matricial no Núcleo de Apoio à Saúde da } \\
\text { Família }\end{array}$ & Klein e Oliveira ${ }^{47}$ & São Paulo-SP & $\begin{array}{l}15 \text { psicólogos } \\
\text { vinculados aos Nasf }\end{array}$ & Qualitativa & 2017 \\
\hline$\stackrel{\bullet}{\mathscr{m}}$ & $\begin{array}{l}\text { Matrix support in mental health in Primary } \\
\text { Health Care: barriers and facilitating factors }\end{array}$ & Hirdes e Silva ${ }^{48}$ & Porto Alegre-RS & $\begin{array}{l}6 \text { trabalhadores de } \\
\text { matriciamento }\end{array}$ & Qualitativa & 2017 \\
\hline$\widehat{\omega}$ & $\begin{array}{c}\text { Apoio Matricial em Saúde Mental no } \\
\text { SUS de Belo Horizonte: perspectiva dos } \\
\text { trabalhadores }\end{array}$ & $\begin{array}{l}\text { Dantas e } \\
\text { Passos }\end{array}$ & $\begin{array}{l}\text { Belo Horizonte- } \\
\qquad \text { MG }\end{array}$ & $\begin{array}{l}7 \text { trabalhadores de } \\
\text { matriciamento }\end{array}$ & Qualitativa & 2018 \\
\hline$\stackrel{\infty}{\tilde{m}}$ & $\begin{array}{l}\text { Apoio matricial em Saúde Mental na atenção } \\
\text { básica: efeitos na compreensão e manejo } \\
\text { por parte de agentes comunitários de saúde }\end{array}$ & Amaral et al ${ }^{50}$ & Salvador-BA & $\begin{array}{l}12 \text { agentes comunitários } \\
\text { de Saúde da } A B\end{array}$ & Qualitativa & 2018 \\
\hline
\end{tabular}

Como pode ser observado no quadro 1 , os estudos rastreados se caracterizam por terem sido publicados majoritariamente no ano de $2012(21 \%-n=8), 2013$ $(15,8 \%-n=6), 2011(13,2 \%-n=5)$ e $2016(13,2 \%-n=5)$. Tratavam-se em sua grande maioria de estudos qualitativos $(92,1 \%-n=35)$, havendo somente três $(8 \%)$ 
estudos mistos e nenhum estudo quantitativo. Envolveram de dois a 137 participantes, havendo 16 (42\%) estudos com até dez participantes, 16 estudos (42\%) com 11 a trinta participantes e seis $(15,8 \%)$ estudos com mais de trinta participantes. A realização dos estudos se deu principalmente na região sudeste do país $(50 \%-n=19)$, seguida da região nordeste $(31,6 \%-n=12)$, sul $(15,8 \%-n=6)$ e centro-oeste $(2,6 \%-n=1)$. Nenhum dos estudos havia sido conduzido na região norte.

Por meio da leitura dos estudos, foram identificados 25 itens relativos a impasses ou desafios para consolidação e efetividade do Apoio Matricial em Saúde Mental na Atenção Básica. Desses itens, nove foram alocados na categoria referente a "obstáculos estruturais", constituindo esta como a categoria com maior número de itens identificados. Destaca-se ainda a categoria "obstáculos subjetivos e culturais" com cinco itens, seguida das categorias "obstáculos decorrentes do excesso de demanda e da carência de recursos" e "obstáculos epistemológicos”, com quatro itens cada, e, por fim, a categoria "obstáculos políticos e de comunicação", com três itens. Cada um dos impasses/desafios identificados por meio dos estudos de acordo com sua alocação nas categorias adotadas está disposto na tabela 1 .

Tabela 1. Impasses e desafios para consolidação e efetividade do apoio matricial na atenção básica identificados nos estudos selecionados

\begin{tabular}{|c|c|}
\hline \multicolumn{2}{|l|}{ Obstáculos estruturais } \\
\hline Impasse/desafio & Estudos que abordam o tema \\
\hline Fragmentação da rede com baixa integração ou burocratização do fluxo entre serviços. & E3; E4; E8; E11; E16; E19; E20; E21; E23; E28; E34. \\
\hline $\begin{array}{l}\text { Perpetuação da lógica do encaminhamento, sem continuidade das ações entre os níveis } \\
\text { de atenção e com baixa responsabilização pelo seguimento dos pacientes. }\end{array}$ & $\begin{array}{l}\text { E1; E2; E4; E5; E8; E9; E11;E13; E20; E21; E23; E24; } \\
\text { E27;E29;E31;E35;E37; E38. }\end{array}$ \\
\hline Falta de uniformidade na estruturação dos serviços. & E11; E12; E33; E34; E35. \\
\hline Inexistência/deficiências dos serviços de informação. & E14; E30; E34. \\
\hline Burocratização do processo de trabalho por meio dos instrumentos de gestão. & E16; E21; E36. \\
\hline Alta rotatividade dos profissionais. & E3; E4; E9; E16; E24; E27; E28; E36; E37. \\
\hline Falta de clareza dos profissionais quanto ao papel do serviço e de sua atuação neste. & E1; E2; E6; E7; E8; E10; E16; E24; E25; E26; E29; E35. \\
\hline Negligência das questões relativas ao território em que os usuários estão inseridos. & E8; E13; E15; E17; E19; E24; E27; E29; E30; E32; E36. \\
\hline Dificuldades de acesso aos serviços por parte dos usuários. & E7; E8; E20; E34. \\
\hline \multicolumn{2}{|l|}{ Obstáculos subjetivos e culturais } \\
\hline Impasse/desafio & Estudos que abordam o tema \\
\hline $\begin{array}{l}\text { Dificuldade/negativa dos profissionais em lidar com questões relacionadas à saúde } \\
\text { mental. }\end{array}$ & E11; E15; E17; E20; E21; E25; E26; E32; E34; E36; E38. \\
\hline Tratamento médico/fármaco-centrado. & $\begin{array}{c}\text { E1; E2; E9; E11; E12; E15; E18; E19; E22; E24; E25; E27; } \\
\text { E29; E31. }\end{array}$ \\
\hline Predomínio de uma visão fármaco-dependente entre os usuários. & E19; E22. \\
\hline Dificuldade em realizar ações intersetoriais. & E9; E13; E24; E31; E32. \\
\hline Distanciamento/desprestígio entre as categorias profissionais. & $\begin{array}{c}\text { E7; E9; E11; E12; E13; E14; E15; E24; E27; E29; E36; } \\
\text { E37. }\end{array}$ \\
\hline \multicolumn{2}{|c|}{ Obstáculos decorrentes do excesso de demanda e da carência de recursos } \\
\hline Impasse/desafio & Estudos que abordam o tema \\
\hline Precarização da estrutura física dos serviços. & E1; E4; E6; E24; E27. \\
\hline $\begin{array}{c}\text { Incompatibilidade da demanda de produtividade do profissional com a lógica proposta } \\
\text { pelos serviços. }\end{array}$ & E16; E21; E36. \\
\hline Falta de profissionais e equipes incompletas. & E2; E6; E7; E14; E26; E31. \\
\hline Sobrecarga dos serviços/excesso de demandas. & E16; E20; E21; E24; E27; E30; E31; E32. \\
\hline
\end{tabular}


Tabela 1. Impasses e desafios para consolidação e efetividade do apoio matricial na atenção básica identificados nos estudos selecionados

\begin{tabular}{|c|c|}
\hline \multicolumn{2}{|l|}{ Obstáculos epistemológicos } \\
\hline Impasse/desafio & Estudos que abordam o tema \\
\hline $\begin{array}{l}\text { Falta de delineamentos claros sobre estratégias para prática do matriciamento, } \\
\text { coordenação dos casos e seguimento longitudinal. }\end{array}$ & E9; E13; E16; E18; E24; E28; E34; E35. \\
\hline $\begin{array}{l}\text { Falta de conhecimentos/habilidades/formação específicos para o desenvolvimento do } \\
\text { trabalho a ser realizado. }\end{array}$ & $\begin{array}{c}\text { E1; E2; E4; E5; E6; E8; E9; E10; E11; E17; E19; E20; E21; } \\
\text { E24; E25; E26; E29; E30; E31; E38. }\end{array}$ \\
\hline $\begin{array}{c}\text { Perpetuação do entendimento biológico sobre o processo saúde-doença em detrimento } \\
\text { do olhar biopsicossocial com centralidade do processo de trabalho no modelo } \\
\text { curativista e individual. }\end{array}$ & $\begin{array}{c}\text { E2; E11; E13; E15; E17; E20; E22; E24; E25; E27; E29; } \\
\text { E30; E34; E36. }\end{array}$ \\
\hline Compartimentalização das ações em saúde em especialidades. & $\begin{array}{l}\text { E1; E2; E11; E13; E15; E19; E20; E21; E25; E26; E27; } \\
\text { E29; E31; E35; E36; E38. }\end{array}$ \\
\hline \multicolumn{2}{|l|}{ Obstáculos políticos e de comunicação } \\
\hline Impasse/desafio & Estudos que abordam o tema \\
\hline $\begin{array}{l}\text { Suscetibilidade e variação das condições diretivas e investimentos conforme as } \\
\text { decisões políticas dos atores municipais e comissionamento dos cargos de gestão. }\end{array}$ & E28; E30; E33; E35; E36; E37. \\
\hline $\begin{array}{l}\text { Modelo de gestão verticalizado com dificuldade de comunicação entre os profissionais } \\
\text { e a gestão. }\end{array}$ & E19; E20; E29; E30; E33; E36. \\
\hline Deficiência ou inexistência de serviço de contrarreferência. & E1; E8; E11; E13; E16; E20; E21; E28. \\
\hline
\end{tabular}

\section{Discussão}

As propostas para o estabelecimento de uma maior integração entre os serviços especializados e as equipes de atenção primária constituem uma tendência em diversos países do ocidente ${ }^{51}$. Além de países como Reino Unido, Irlanda e Espanha, cabe destaque para o contexto canadense e australiano, onde são registradas iniciativas voltadas especialmente para qualificação do cuidado em saúde mental ${ }^{52,53}$.

Nesses contextos, o cuidado compartilhado/colaborativo é definido pela participação conjunta de profissionais da atenção primária e especialistas no planejamento dos projetos terapêuticos. Suas práticas são mediadas por arranjos organizacionais que visam ao estabelecimento do papel de coordenação dos casos pela Atenção Primária, a introdução de mecanismos para vinculação entre os profissionais e o desenvolvimento de estratégias para coletar e compartilhar informação sobre o progresso dos usuários ${ }^{51}$.

A rigor, a proposta de apoio matricial brasileira vai além e incorpora ainda o compromisso com a construção de relaçôes democráticas a partir da cogestão e da construção compartilhada do cuidado. Nesse sentido, é previsto que os serviços facilitem as relaçóes comunicativas e dialógicas entre os profissionais de saúde e, sobretudo, entre estes e os usuários, para que os últimos possam participar ativamente da elaboração dos seus projetos terapêuticos ${ }^{51}$.

Contudo, tanto no contexto brasileiro quanto no internacional, parece não estarem claros quais seriam os aspectos imprescindíveis para a efetividade desses arranjos, ocasionando uma grande variedade de conteúdo e intensidade destes. Embora essas variaçôes tenham relação com o aspecto de plasticidade, essencial para adaptação da proposta em diferentes cenários, os resultados deste estudo apontam para uma falta de clareza dos profissionais quanto ao papel dos serviços aos quais estão vinculados e quanto à sua atuação em tais serviços. 
Ressalta-se que, dos 38 estudos selecionados, 12 traziam esse aspecto em seus resultados e, ao contrário do que se poderia supor, não se tratam apenas de estudos relativos ao período mais inicial da proposta, mas sim estudos pulverizados ao longo dos anos, cujas publicações abrangem desde o ano de 2009 a 2017. Dessa forma, ao levar em conta os dez anos do estabelecimento do Nasf como vetor do apoio matricial nas redes de saúde, evidencia-se a necessidade avaliar esse processo, buscando estabelecer prioridades para as agendas de pesquisa e intervenção a partir desses serviços.

Nesse sentido, as próximas sessóes discutem os impasses e desafios para a consolidação e efetividade do apoio matricial em saúde mental de acordo com sua categoria, a saber: obstáculos estruturais, obstáculos subjetivos e culturais, obstáculos decorrentes do excesso de demanda e da carência de recursos, obstáculos epistemológicos e obstáculos políticos e de comunicação.

\section{Obstáculos estruturais}

Para além da falta de clareza dos profissionais quanto ao papel dos serviços e quanto à sua atuação, do ponto de vista estrutural, cabe ainda destacar a falta de uniformidade na estruturação dos serviços, citada por cinco dos estudos rastreados. Ressalta-se que não se tratam apenas de desigualdades regionais, já que, em alguns casos, essas desigualdades são observadas dentro do mesmo município.

Embora a proposta de apoio matricial preveja certa plasticidade que permita aos serviços se adequarem às disparidades de cada território, parece não ser essa a questão que permeia as desigualdades no desempenho da proposta. Cabe destacar que a negligência da territorialização foi citada por 11 dos estudos rastreados.

Nesse sentido, assim como no estudo de Starfield ${ }^{54}$ conduzido no Reino Unido, os dados levantados chamam atenção para a necessidade de se esclarecer o papel dos profissionais - especialistas e generalistas - dentro da proposta. Semelhante ao encontrado neste estudo, Starfield ${ }^{54}$ indica que a inserção dos especialistas como suporte para atenção primária acontecia de forma variável, abrangendo desde consultas breves e intervençôes pontuais à adoção total dos cuidados de alguns usuários.

Além da definição clara dos papéis de cada serviço dentro da proposta, é imprescindível que sua operacionalização seja viabilizada. Nesse sentido, destaca-se que o apoio matricial pressupóe o compartilhamento dos casos e a corresponsabilização pelo cuidado dos usuários. Contudo, a fragmentação da rede com baixa integração ou burocratização do fluxo entre os serviços, citada por 11 dos estudos rastreados, apresenta-se como uma dificuldade para o alcance desses pressupostos. Como resultado deste cenário, tem-se, além de uma perpetuação da lógica do encaminhamento, a falta de continuidade das açóes entre os níveis de atenção, evidenciada por 18 dos estudos rastreados.

Por fim, cabe destacar que tanto a proposta de territorialização quanto a demanda de corresponsabilização pelo cuidado dos usuários só pode ser viabilizada pelo estabelecimento de vínculo entre os trabalhadores e o local onde atuam e a sua clientela. Contudo, os resultados deste estudo indicam a persistência de uma alta rotatividade de profissionais vinculados tanto aos serviços de matriciamento quanto aos serviços de Atenção Primária. Esse aspecto esteve presente em nove dos 
estudos rastreados e diz respeito a uma realidade incompatível com a proposta de matriciamento. A necessidade de um plano claro e definido para contratação dos profissionais, com previsão de estratégias de capacitação e fixação, já foi apontada na perspectiva do cuidado compartilhado em saúde mental na Austrália. Kelly et a ${ }^{53}$ defendem que esse é um dos "ingredientes principais" para a efetivação desse tipo de proposta.

\section{Obstáculos subjetivos e culturais}

Outro aspecto importante levantado por Kelly et al ${ }^{53}$, no que diz respeito à necessidade de um plano claro e definido para contratação dos profissionais, tem relação com a aptidão e disposição desses profissionais para o trabalho em saúde mental. Ressalta-se que a resistência por parte de alguns profissionais para o cuidado ao usuário com transtornos mentais e necessidades em saúde mental ainda é muito presente no país. Embora nas últimas décadas tenha-se avançado muito do ponto de vista político, poucas ações foram direcionadas para fora dos serviços no que diz respeito ao enfrentamento do estigma vivenciado por essa população ${ }^{55}$.

Para além dessa realidade, não se pode deixar de levar em conta que os indivíduos possuem aspirações profissionais próprias e maior identificação com determinadas áreas de sua profissão. Nesse sentido, é importante que se estabeleçam processos seletivos claros, direcionados e sensíveis à captação de profissionais qualificados para trabalho em saúde mental em detrimento aos processos que vêm comumente acontecendo no país. São frequentes os editais que preveem a contratação de trabalhadores baseados apenas em suas categorias profissionais para subsequente alocação em algum serviço, à revelia das aptidóes do candidato.

O desfecho dessa perspectiva pode ser observado nos resultados deste estudo, uma vez que 11 dos estudos rastreados citaram a dificuldade ou negativa dos profissionais em lidar com as questóes relacionadas à saúde mental. Ressalta-se que não se trata uma realidade restrita aos serviços da atenção primária, havendo relatos mesmo entre profissionais de serviços especializados.

Outro aspecto que pode ter uma relação com essa perspectiva é a perpetuação de um tratamento médico e/ou fármaco-centrado. Embora dois estudos relatem que os próprios usuários muitas vezes apresentam uma demanda fármaco-dependente, 14 estudos pontuaram o predomínio de práticas medicalizantes tanto nos serviços de atenção primária quanto nos serviços especializados.

Por fim, cabe destacar como um importante obstáculo subjetivo o distanciamento e/ou desprestígio entre as categorias profissionais. Este obstáculo foi recorrente em 12 dos estudos rastreados e se reflete em diversas situaçóes, cabendo destaque para aquelas em que determinadas categorias profissionais, como os agentes comunitários de saúde, são desconsideradas nas discussões dos casos e em situações em que o matriciamento é visto como uma atividade secundária e de menor importância. Neste último caso, são recorrentes os relatos de esvaziamento das reunióes de matriciamento e ausência de categorias específicas, em especial a médica.

Nesse sentido, cabe destacar que, na meta-análise realizada por Foy et al $^{56}$ sobre o cuidado compartilhado, a comunicação interprofissional emergiu como um importante fator associado à efetividade do modelo. Para os autores, é importante que 
as equipes criem coordenadas organizacionais a fim de superar as dificuldades para realização de encontros, manifestadas, por exemplo, pela falta de tempo nas agendas. Dessa forma, seria garantida a possibilidade de comunicação interativa entre os profissionais e sua consequente aproximação.

\section{Obstáculos decorrentes do excesso de demanda e da carência de recursos}

Como pontuado anteriormente, é imprescindível que os serviços sejam viáveis à execução da proposta de apoio matricial para que ele possa de fato ser efetivado. Isso inclui a garantia de recursos mínimos para operacionalização da proposta, como locais adequados para as reuniôes de equipes e atendimento dos usuários, número adequado de profissionais e compatibilidade das demandas de atendimento e das atividades-meio com a capacidade de cada equipe.

Apesar de uma suposta clareza quanto a essas questóes, foram recorrentes nos estudos rastreados aspectos que vão na contramão desse entendimento. Destaca-se em especial o excesso de demandas que têm sobrecarregado os serviços, tanto de atenção primária quanto especializados, tendo sido citado em oito dos estudos rastreados.

\section{Obstáculos epistemológicos}

Os resultados deste estudo indicaram uma forte prevalência de obstáculos epistemológicos para consolidação e efetividade do apoio matricial em saúde mental. Dentre esses obstáculos, destacam-se dois fatores que são relacionados às condiçôes estruturantes da proposta: a falta de delineamentos claros sobre as estratégias para prática do matriciamento; coordenação dos casos e seguimento longitudinal; e a falta de conhecimentos e/ou habilidades e/ou formação específicas para o desenvolvimento do trabalho a ser realizado. O primeiro foi citado por oito dos estudos rastreados e o segundo, por vinte estudos.

Dada a persistência desses obstáculos, não é inesperado que uma série de outras fragilidades e desdobramentos negativos esteja presente. Destaca-se que a definição clara do arranjo e a formação para sua operacionalização são recorrentes nos estudos internacionais que tratam de metodologias semelhantes, seja na perspectiva do cuidado colaborativo ou compartilhado.

Kelly et $\mathrm{al}^{53}$, por exemplo, destaca como necessidade para efetivação da proposta de cuidado compartilhado em saúde mental na Austrália o estabelecimento de um acordo para a definição do modelo clínico e do monitoramento dos pacientes. Uma perspectiva semelhante é apontada por Foy et al ${ }^{56}$. Para esses autores, é indispensável que se pactuem as coordenadas organizacionais para as equipes que trabalham nessa lógica. Nesse sentido, Starfield ${ }^{54}$ aponta ainda para a demanda de definir claramente o papel dos profissionais, generalistas e especialistas, no arranjo.

Ressalta-se que o delineamento claro das estratégias de matriciamento e do papel dos profissionais é o primeiro passo para orientar as açóes de formação e capacitação dos profissionais. Nesse sentido, para além de investimentos maciços na formação dos profissionais da saúde que trabalharão segundo a lógica desse cuidado, incluindo mudanças nos currículos de graduação e de residência, é necessário que se estabeleçam 
ações de educação permanente para capacitação de profissionais já inseridos nos serviços.

Conforme apontado por Kates e Craven ${ }^{57}$ ao avaliarem a perspectiva do cuidado compartilhado em saúde mental no Canadá, os gestores em geral têm começado a aceitar os princípios dessa lógica de atenção. Contudo, somente a partir de uma aproximação estratégica com os profissionais da ponta questóes como a perpetuação do entendimento biológico sobre o processo saúde-doença em detrimento do olhar biopsicossocial (citada por 14 dos estudos rastreados) e a compartimentalização das ações de saúde em especialidades (citada por 16 dos estudos rastreados) serão superadas.

\section{Obstáculos políticos e de comunicação}

A aproximação dos profissionais diretamente ligados à assistência dos usuários com ofertas sistemáticas de capacitação e supervisão, além de potente reorientadora das práticas, é estratégica para sustentabilidade da proposta. Ressalta-se que um ponto importante a ser considerado é a instabilidade do contexto político sanitário brasileiro, historicamente associada à descontinuidade administrativa e aos dilemas, práticas e contradiçóes que surgem na administração pública a cada mudança de governo e troca de dirigentes ${ }^{58}$.

Destaca-se que a suscetibilidade e variação das condiçôes diretivas e dos investimentos conforme a decisão política dos atores municipais foi um aspecto evidenciado nos resultados deste estudo. Da mesma forma, surgiram ainda as questóes relativas ao comissionamento dos cargos de gestão, aspecto que dificulta o seguimento das ações por meio da alternância de governos e, em alguns casos, atribui o papel de gestão do arranjo a indivíduos sem competência técnica para tal. Indicativos dessa realidade foram evidenciados por seis dos estudos rastreados.

Cabe destacar ainda os problemas relativos à comunicação, em alguns casos relacionados aos modelos verticalizados de gestão (conforme citado por seis dos estudos rastreados), e, em outros, relacionados à deficiência ou inexistência de serviços de contrarreferência (conforme citado por oito dos estudos rastreados). Em ambos os casos, tratam-se de situaçóes que inviabilizam a efetividade da proposta, uma vez que, assim como pontuado por Vingilis et al..$^{52}$ ao avaliar os processos de um programa de cuidado colaborativo no Canadá, a comunicação é um ponto-chave para a construção conjunta dos projetos terapêuticos, bem como para o estabelecimento de soluçóes aos entraves na operacionalização do arranjo.

Vingilis et $\mathrm{a}^{52}$ advogam que um dos elementos para o sucesso do cuidado colaborativo seria o que os trabalhadores acessados chamam de "política de portas abertas". Nesse modelo, todos os profissionais sabem onde os generalistas e especialistas estão alocados e têm liberdade para procurá-los nos momentos em que forem mais oportunos para o tratamento de algum caso. Já Kelly et $\mathrm{al}^{53}$, ao tratarem do cuidado compartilhado em saúde mental na Austrália, citam a necessidade da provisão de encontros sistemáticos para revisão dos casos como um dos elementos para esse tipo de trabalho. Nesse sentido, independente do tipo de logística adotada, fica evidente a importância de superar as dificuldades de comunicação a fim de alcançar a efetividade desse tipo de arranjo. 


\section{Considerações finais}

Passados dez anos da promulgação da portaria que institui o apoio matricial nas redes de saúde por meio do Nasf, fica evidente o crescimento das produçóes acerca da temática. Contudo, os resultados deste estudo sugerem que ainda é preciso avançar nos estudos de avaliação dessa política, especialmente a partir dos estudos quantitativos e mistos. Para isso, recomenda-se que os pesquisadores busquem definir um quadro comum de indicadores para avaliação da proposta, bem como lançar mão de desenhos cada vez mais participativos, tanto em amplitude quanto em profundidade.

Destaca-se que os impasses e desafios para consolidação e efetividade do apoio matricial em saúde mental encontrados neste estudo se relacionam, em sua grande maioria, com resultados encontrados nos estudos internacionais acerca dos congêneres dessa proposta.

Nesse sentido, entre os aspectos que precisam ser levados em conta na qualificação dos serviços já implementados, bem como na implementação de novos serviços, sugere-se: a) Definição de delineamentos para prática de matriciamento, coordenação dos casos e seguimento longitudinal com atribuição clara do papel dos profissionais da atenção primária e especialistas; b) Investimento maciço em formação e capacitação dos profissionais, especialmente aqueles envolvidos diretamente na prestação dos cuidados, para atuar na lógica do compartilhamento e corresponsabilização dos casos; e c) criação de espaços institucionalizados com encontro sistemático dos profissionais para discussão dos casos e avaliação conjunta do andamento das atividades.

\section{Contribuições dos autores}

Todos os autores participaram ativamente de todas as etapas de elaboração do manuscrito.

\section{Agradecimentos}

À Coordenação de Aperfeiçoamento de Pessoal de Nível Superior (Capes) pelo financiamento parcial desta pesquisa por meio de bolsa de estudos concedida ao autor principal deste artigo - Código de financiamento 001.

\section{Direitos autorais}

Este artigo está licenciado sob a Licença Internacional Creative Commons 4.0, tipo BY (https://creativecommons.org/licenses/by/4.0/deed.pt_BR).

$(\mathrm{cc}) \boldsymbol{B}^{\mathrm{B}}$

\section{Referências}

1. Cook JA, Razzano LA, Swarbrick MA, Jonikas JA, Yost C, Burke L, et al. Health risks and changes in self-efficacy following community health screening of adults with serious mental illnesses. PloS One. 2015; 10(4):e0123552. 
2. Organização Mundial da Saúde. MhGAP intervention guide for mental, neurological and substance use disorders in non-specialized health settings: Mental Health Gap Action Programme (mhGAP). Genebra: OMS; 2010.

3. Thornicroft $\mathrm{G}$, Tansella $\mathrm{M}$. What are the arguments for community-based mental health care? Copenhagen: WHO Regional Office for Europe; 2003.

4. Paulon S, Neves R. Saúde mental na atenção básica, a territorialização do cuidado. Porto Alegre: Sulina; 2013.

5. Onocko-Campos RT, Gama CA, Ferrer AL, Santos DVD, Stefanello S, Trapé TL, et al. Saúde mental na atenção primária à saúde: estudo avaliativo em uma grande cidade brasileira. Cienc Saude Colet. 2011; 16(12):4643-52.

6. Onocko-Campos RT, Campos GWS, Ferrer AL, Corrêa CRS, Madureira PR, Gama $\mathrm{CAP}$, et al. Avaliação de estratégias inovadoras na organização da atenção primária à saúde. Rev Saude Publica. 2012; 46(1):43-50.

7. Hirdes A. A perspectiva dos profissionais da atenção primária à saúde sobre o apoio matricial em saúde mental. Cienc Saude Colet. 2015; 20(2):371-82.

8. Athié K, Menezes AL, Silva AM, Campos M, Delgado PG, Fortes S, et al. Perceptions of health managers and professionals about mental health and primary care integration in Rio de Janeiro: a mixed methods study. BMC Health Serv Res. 2016; 30(1):532.

9. Bower P, Gilbody S, Richards D, Fletcher J, Sutton A. Collaborative care for depression in primary care. Making sense of a complex intervention: systematic review and meta-regression. Br J Psychiatry. 2006; 189:484-93.

10. Smith SM, Allwright S, O'Dowd T. Effectiveness of shared care across the interface between primary and specialty care in chronic disease management. Cochrane Database Syst Rev. 2007; (3):CD004910.

11. Campos GWS. Equipes de referência e apoio especializado matricial: um ensaio sobre a reorganização do trabalho em saúde. Cienc Saude Colet. 1999; 4(2):393-403.

12. Castro CP, Oliveira MM, Campos GWS. Apoio matricial no SUS Campinas: análise da consolidação de uma prática interprofissional na rede de saúde. Cienc Saude Colet. 2016; 21(5):1625-36.

13. Brasil. Ministério da Saúde. Portaria 154, de 24 de Janeiro de 2008. Dispóe sobre a criação dos Núcleos de Apoio à Saúde da Família. Diário Oficial da União. 4 Mar 2008.

14. Brasil. Secretaria de Tecnologia da Informação. Distribuição dos Núcleos de Apoio à Saúde da Família - NASF. Brasília: Ministério do Planejamento, Desenvolvimento e Gestão; 2018 [citado 13 Out 2018]. Disponível em: http://dados.gov.br/dataset/ nasf_12

15. Campos GWS, Domitti AC. Apoio matricial e equipe de referência: uma metodologia para gestão do trabalho interdisciplinar em saúde. Cad Saude Publica. 2007; 23(2):399-407.

16. Minayo MCS. O desafio do conhecimento: pesquisa qualitativa em saúde. 5 a ed. São Paulo: Hucitec, Abrasco; 1998.

17. Dimenstein M, Severo AK, Brito M, Pimenta AL, Medeiros V, Bezerra E. O apoio matricial em unidades de saúde da família: experimentando inovaçóes em saúde mental. Saude Soc. 2009; 18(1):63-74.

18. Figueiredo MD, Onocko-Campos RT. Saúde mental na atenção básica à saúde de Campinas, SP: uma rede ou um emaranhado? Cienc Saude Colet. 2009; 14(1):129-38. 
19. Mielke FB, Olchowsky A. Saúde mental na estratégia saúde da família: a avaliação de apoio matricial. Rev Bras Enferm. 2010; 63(6):900-7.

20. Cossetin A, Olschowsky A. Avaliação das ações em saúde mental na estratégia de saúde da família: necessidades e potencialidades. Rev Gauch Enferm. 2011; 32(3):495-501.

21. Ballarin MLGS, Carvalho FB, Ferigato SH, Miranda IMS, Magaldi CC. Centro de atenção psicossocial: convergência entre saúde mental e coletiva. Psicol Estud. 2011; 16(4):603-11.

22. Silva DS. Apoio matricial em saúde mental: uma análise sob ótica dos profissionais de saúde da atenção primária. Rev Port Enferm Saude Mental. 2011; (6):20-7.

23. Sousa FSP, Jorge MSB, Vasconcelos MGF, Barros MMM, Quinderé PHD, Gondim LGF. Tecendo a rede assistencial em saúde mental com a ferramenta matricial. Physis. 2011; 21(4):1579-99.

24. Ballarin MLGS, Blanes LS, Ferigato SE. Apoio matricial: um estudo sobre a perspectiva de profissionais da saúde mental. Interface (Botucatu). 2012; 16(42):76778.

25. Cavalcante CM, Jorge MSB, Santos DCM. Onde está a criança?: desafios e obstáculos ao apoio matricial de crianças com problemas de saúde mental. Physis. 2012; 22(1):161-78.

26. Jorge MSB, Pinto DM, Vasconcelos MGF, Pinto AGA, Souza RS, Caminha ECCR. Ferramenta matricial na produção do cuidado integral na estratégia saúde da família. Acta Paul Enferm. 2012; 25 n esp 2:26-32.

27. Delfini PSS, Reis AOA. Articulação entre serviços públicos de saúde nos cuidados voltados à saúde mental infantojuvenil. Cad Saude Publica. 2012; 28(2):357-66.

28. Morais APP, Tanaka OY. Apoio matricial em saúde mental: alcances e limites na atenção básica. Saude Soc. 2012; 21(1):161-70.

29. Rodrigues ES, Moreira MIB. A interlocução da saúde mental com atenção básica no município de Vitoria/ES. Saude Soc. 2012; 21(3):599-611.

30. Vannucchi AMC, Carneiro JN. Modelos tecnoassistenciais e atuação do psiquiatra no campo da atenção primária à saúde no contexto atual do Sistema Único de Saúde, Brasil. Physis. 2012; 22(3):963-82.

31. Freire FMS, Pichelli AAWS. O psicólogo apoiador matricial: percepções e práticas na atenção básica. Psicol Cienc Prof. 2013; 33(1):162-73.

32. Minozzo F, Costa II. Apoio matricial em saúde mental: fortalecendo a saúde da família na clínica da crise. Rev Latinoam Psicopatol Fundam. 2013; 16(3):438-50.

33. Leite $\mathrm{DC}$, Andrade $\mathrm{AB}$, Bosi MLM. A inserção da psicologia nos núcleos de apoio à saúde da família. Physis. 2013; 23(4):1167-87.

34. Quinderé PHD, Jorge MSB, Nogueira MSL, Costa LFA, Vasconcelos MGF.

Acessibilidade e resolubilidade da assistência em saúde mental: a experiência do apoio matricial. Cienc Saude Colet. 2013; 18(7):2157-66.

35. Minozzo F, Costa II. Apoio matricial em saúde mental entre CAPS e saúde da família: trilhando caminhos possíveis. Psico-USF. 2013; 18(1):151-60.

36. Jorge MSB, Sousa FSP, Franco TB. Apoio matricial: dispositivo para resolução de casos clínicos de saúde mental na atenção primária à saúde. Rev Bras Enferm. 2013; 66(5):738-44. 
37. Gazignato ECS, Silva CRC. Saúde mental na atenção básica: o trabalho em rede e o matriciamento em saúde mental na estratégia de saúde da família. Saude Debate. 2014; 38(101):296-304.

38. Hori AA, Nascimento AF. O projeto terapêutico singular e as práticas de saúde mental nos núcleos de apoio à saúde da família (NASF) em Guarulhos (SP), Brasil. Cienc Saude Colet. 2014; 19(8):3561-71.

39. Pegoraro RF, Cassimiro TJL, Leão NC. Matriciamento em saúde mental segundo profissionais da estratégia da saúde da família. Psicol Estud. 2014; 19(4):621-31.

40. Costa FRM, Lima VV, Silva RF, Fioroni LN. Desafios do apoio matricial como prática educacional: a saúde mental na atenção básica. Interface (Botucatu). 2015; $19(54): 491-502$.

41. Jorge MSB, Diniz AM, Lima LL, Penha JC. Apoio matricial, projeto terapêutico singular e produção do cuidado em saúde mental. Texto Contexto Enferm. 2015; 24(1):112-20.

42. Iglesias A, Avellar SZ. As contribuições dos psicólogos para o matriciamento em saúde mental. Psicol Cienc Prof. 2016; 36(2):364-79.

43. Reis ML, Medeiros M, Pacheco LR, Caixeta CC. Avaliação do trabalho multiprofissional do núcleo de apoio à saúde da família (NASF). Texto Contexto Enferm. 2016; 25(1):e2810014.

44. Salvador DB, Pio DAM. Apoio matricial e Capsi: desafios do cenário na implantação do matriciamento em saúde mental. Saude Debate. 2016; 40(111):246-56.

45. Lima M, Dimenstein M. O apoio matricial em saúde mental: uma ferramenta apoiadora da atenção à crise. Interface (Botucatu). 2016; 20(58):625-35.

46. Soares S, Oliveira WF. The matrix approach to mental health care: experiences in Florianopolis, Brazil. J Health Psychol. 2016; 21(3):336-45.

47. Klein AP, d'Oliveira AFPL. O “cabo de força” da assistência: concepção e prática de psicólogos sobre o apoio matricial no núcleo de apoio à saúde da família. Cad Saude Publica. 2017; 33(1):e00158815.

48. Hirdes A, Silva MKR. Matrix support in mental health in primary health care: barriers and facilitating factors. Estud Psicol. 2017; 34(4):499-511.

49. Dantas NF, Passos ICF. Apoio matricial em saúde mental no SUS de Belo Horizonte: perspectiva dos trabalhadores. Trab Educ Saude. 2018; 16(1):201-20.

50. Amaral CEM, Torrenté MON, Torrenté M, Moreira CP. Apoio matricial em saúde mental na atenção básica: efeitos na compreensão e manejo por parte de agentes comunitários de saúde. Interface (Botucatu). 2018; 22(66):801-12.

51. Oliveira MM, Campos GWS. Apoios matricial e institucional: analisando suas construçốes. Cienc Saude Colet. 2015; 20(1):229-38.

52. Vingilis E, Paquete-Warren J, Kates N, Crustolo A, Greenslade J, Newman S. Descriptive and process evaluation of a shared primary care program. Internet J Allied Health Sci Pract. 2007; 5(4):1-10.

53. Kelly BJ, Perkins DA, Fuller JD, Parker SM. Shared care in mental illness: a rapid review to inform implementation. Int J Ment Health Syst. 2011; 5:31.

54. Starfield B. William Pickles lecture. Primary and specialty care interfaces: the imperative of disease continuity. Br J Gen Pract. 2003; 53(494):723-9. 
55. Nunes $M$, Torrenté $M$. Estigma e violências no trato com a loucura: narrativas de centros de atenção psicossocial, Bahia e Sergipe. Rev Saude Publica. 2009; 43 Suppl 1:101-8.

56. Foy R, Hempel S, Rubenstein L, Suttorp M, Seelig M, Shanman R, et al. Metaanalysis: effect of interactive communication between collaborating primary care physicians and specialists. Ann Intern Med. 2010; 152(4):247-58.

57. Kates N, Craven M, Collaborative Working Group of the College of Family Physicians of Canada, Canadian Psychiatric Association. Shared mental health care. Update from the collaborative working group of the college of family physicians of Canada and the Canadian Psychiatric Association. Can Fam Physician. 2002; 48:936.

58. Nogueira FA. Continuidade e descontinuidade administrativa em governos locais: fatores que sustentam a ação pública ao longo dos anos [tese]. São Paulo: Escola de Administração de Empresas de São Paulo; 2006.

Ten years on from the introduction of matrix support in health networks through the creation of Family Health Support Centers, there is still a lack of consistent data to measure the success of consolidation and effectiveness of this arrangement. With this in mind, we conducted a literature review of national articles produced over the last ten years to identify the bottlenecks and challenges faced by matrix support in mental health in Primary Care. The problems were classified using a theoretical and conceptual reconstruction and drawing on similar experiences with matrix support in other countries. The following key points emerged from the review: the need to provide clear guidelines for matrix support; the need for major investment in training and capacity building; and the need to create institutionalized spaces to foster systematic communication between professionals to discuss cases and promote joint evaluation of the progress of activities.

Keywords: Matrix support. Mental health. Primary healthcare.

Transcurridos diez ańos desde la implementación del apoyo matricial en las redes de salud por medio de los Núcleos de Apoyo a la Salud de la Familia, todavía no hay datos consistentes en lo que se refiere a la consolidación y efectividad de ese arreglo. En ese sentido, este estudio tuvo el objetivo de revisar la bibliografía nacional de los últimos diez años con el objetivo de identificar los impases y desafíos vividos en el apoyo matricial en salud mental en la Atención Primaria, clasificándolos a partir de una reconstrucción teórico-conceptual y haciendo una articulación de ellos con los desafíos puntuados en congéneres internacionales del apoyo matricial. Entre los principales puntos encontrados por el estudio se destacó la necesidad de delineamientos claros para la práctica matricial, inversión maciza en formación y capacitación de los profesionales y creación de espacios institucionalizados con encuentro sistemático de los profesionales para discusión de los casos y evaluación conjunta del curso de las actividades.

Palabras clave: Apoyo matricial. Salud mental. Atención primaria de la salud. 